\title{
Acute Small Gut Obstruction as an Atypical Presentation of Acute Appendicitis without Rupture or Perforation of the Appendix
}

\author{
ABM MONIRUDDIN $^{\mathrm{a}}, \mathrm{S} \mathrm{CHOWDHURY}^{\mathrm{b}}, \mathrm{THASAN}^{\mathrm{c}}, \mathrm{K}^{\mathrm{N}} \mathrm{M} \mathrm{M} \mathrm{HASAN}^{\mathrm{d}}, \mathrm{R} \mathrm{JAHAN}^{\mathrm{e}}, \mathrm{KAHOSSAIN}^{\mathrm{f}}$
}

\begin{abstract}
:
Acute appendicitis is a very common surgical emergency. It has got diverse presentations and various complications. Here, we report case history of a young male patient with a 2year history of recurrent abdominal pain, who presented to our center with a 4-day history of increasing abdominal pain, constipation and vomiting. Clinical and radiological evaluation revealed it as a case of acute intestinal obstruction preoperatively. Per-operative findings suggested acute appendicitis without perforation, small gut obstruction by
\end{abstract}

Introduction:

Acute appendicitis and acute small intestinal obstruction are two different common surgical emergencies having different aetiologies, pathologies and treatment modalities. Appendicitis is the inflammation of the vermiform appendix, usually associated with microbial infection, predisposed and precipitated by a single or combination of several independent factors like luminal obstruction by faecolith, worms, food particles, lymphoid hyperplasia, neoplasms etc. ${ }^{1}$ The pain of acute

a. Dr. ABM Moniruddin MBBS, FCPS(S). Prof of Surgery, Monno Medical College, Manikganj.

b. Dr. Salma Chowdhury MBBS, MCPS(Obs\&Gynae), DGO. Ex-Prof of Obs\&Gynae, Dhaka Mrdical College, Dhaka.

c. Dr. TanvirulHasan MBBS. MO, Institute of Nuclear Medicine \& Allied Sciences, BAEC, Dinajpur.

d. Dr. Kazi Muhammad Mahmudul Hasan MBBS, DA. Assist Prof of Anaesthesiology, Monno Medical College, Manikganj.

e. Dr. RounakJahan MBBS. MO, Department of Surgery, Monno Medical College, Manikganj.

f. Dr. KabirAshfaqueHossain MO, Department of Surgery, Monno Medical College, Manikganj.

Address of Correspondence: ABM Moniruddin. Prof of Surgery, Monno Medical College, Manikganj. Mobile: 008801795078217. E-mail: abmmu@yahoo.com

Received: 16 Sept., 2020

Accepted: 26 June, 2021 adhesions, appendicular lump or internal herniation. Appendicectomy was done along with other necessary procedures. His post-operative period was uneventful. This case is reported here to highlight that acute appendicitis may present with atypical features like acute small gut obstruction.

Keywords: Adhesion, appendicular lump, atypical appendicitis, internal herniation, Ileus.

(J Bangladesh Coll Phys Surg 2021; 39: 261-265)

DOI: https://doi.org/10.3329/jbcps.v39i4.55947

appendicitis usually starts at umbilical or peri-umbilical or epigastric region that soon gets localized in right iliac region. Abnormal position and orientation of the appendix interfering with drainage of its luminal secretion and contents or compromising its blood supply causing ischemia may lead to appendicitis.Histopathologically, pan-inflammatory involvement of all coats and presence of neutrophils in muscular coats are diagnostic. ${ }^{2,3}$ Acute intestinal obstruction having most commonly different pathogenesis is not an immediate sequelae of acute appendicitis. Acute, subacute and chronic intestinal obstructions following acute appendicitis have been identified. ${ }^{4-6}$ The clinical pictures of small gut obstruction may dominate over the clinical features of acute appendicitis, the latter being identified as 'masked acute appendicitis'. Acute appendicitis has been identified as a cause of small gut obstruction. ${ }^{7,8}$ Misdiagnosis delays the specific treatment causing life-threatening complications, increasing morbidity and mortality. Here, we report a case of acute appendicitis who presented with features of intestinal obstruction.

\section{Case Report:}

A 26-year-oldmale presented with intermittent attacks of central abdominal pain for about two years; the current episode started four days before presenting to our center. This time, he had severe abdominal pain, nausea, 
vomiting, constipation and restlessness. For the last two years, the pain had no definite periodicity and no definite relation to food and medicines. He had been then treated locally village physicians without having any laboratory investigations and definite diagnosis. Four days back, he had developed sudden increasing epigastric pain that soon involved the umbilical and left iliac regions. And eventually the pain was more marked in the right iliac region. On the second day, the pain spread to all over the whole abdomen. On the third day, he had noted gradual abdominal distension with feeling of abdominal tightness, respiratory distress, nausea, vomiting, constipation and increasing restlessness. Initially the vomitus contained undigested food particles. Later it was found bile stained. This time he was also treated by local nonqualified village physicians by oral esomeprazole, oral tiemonium and oral antacid suspension without having any improvement. He had no remarkable other past illness. None of his family members suffered from similar type of illnesses.

He was found dehydrated and febrile at presentation. There were no scar marks and no pigmentation on abdomen. His hernial orifices were intact with normal hair distribution. His abdomen was highly tense and highly tender with generalized board-like rigidity suggestive of peritonitis. Deep palpation was not possible. Percussion notes on abdomen were tympanic with no obliteration of liver dullness. Bowel sounds were not audible. Per rectal examination was not contributory.

His total leucocyte count was 12,000 per microL with neutrophils $93 \%$, serum amylase 158 units/L, serum $\mathrm{Na}^{+}$ $156 \mathrm{mmol} / \mathrm{L}$, serum K $4.21 \mathrm{mmol} / \mathrm{L}$, serum $\mathrm{Cl}^{-} 97.9 \mathrm{mmol} /$ L,C-reactive protein was $20 \mathrm{mg} / \mathrm{L}$. No other notable abnormalities were detectable on ultrasonogram, chest $\mathrm{X}$-ray and electrocardiogram (ECG). Blood sample was drawn and sent for culture and sensitivity tests (that later on showed no growth of any microbe). Plain X-ray abdomen revealed centrally placed multiple abnormal shadows of gases and fluid levels (Figure 1) with prominent valvulaeconnivantes suggestive of small gut obstruction and there was no feature of perforation.

After rapid resuscitation, emergency laparotomy was done. We found distended small intestinal loops involving mostly proximal and mid jejunal parts, some of which were adherent to themselves by fibrinous adhesion and to the inflamed highly distended partly gangrenous floating pelvic appendix (Figures 2 and 3).

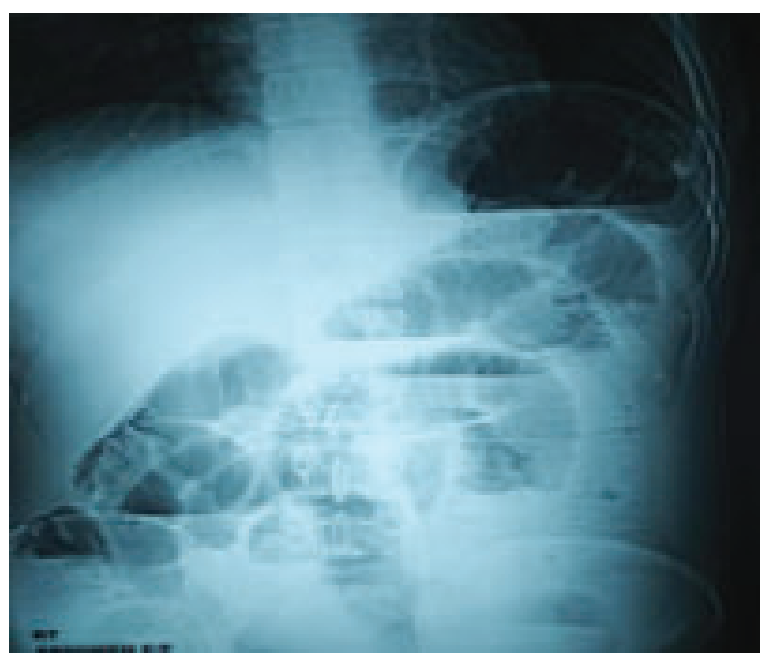

Fig.-1: Plain X-ray abdomen showing features of small gut obstruction
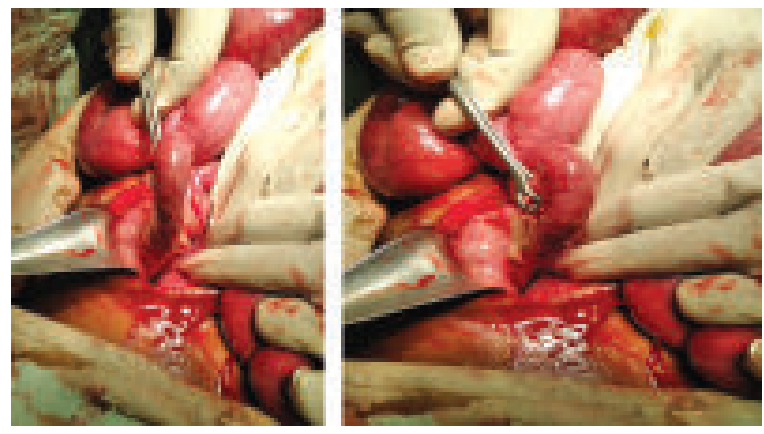

Fig. 2 \& 3: Showing inflamed distended oedematous vermiform appendix with an area of gangrene and its engorged blood vessels and distended small gut

No fibrous adhesion, band, faecal matter, worm or no undigested food particles other than some fibrinous adhesions and inflammatory exudates were found in the inflamed peritoneal cavity (peritonitis). The appendix was found highly distended, inflamed, oedematous, partly gangrenous at nearly its mid-point (and not at its tip) with engorged appendiceal vessels with no evidence of abscess, perforation or rupture/bursting (i.e., no burst appendix). The terminal and mid-ileal segments were found collapsed. Per-operatvely, it was diagnosed as a case acute intestinal obstruction complicating acute appendicitis due to sepsis and peritonitis, complicated by transmigrated microbes, without perforation or 
rupture of the appendix. Appendicectomy was done. That was followed by manual decompression by milking the jejunal contents into the large gut, peritoneal toileting and cleansing to remove inflammatory exudates and fibrinous adhesions, anal stretching, along with intravenous broad spectrum combination antibiotic administration (covering most gram positive, gram negative and anaerobic microbes) for several days. Histopathology revealed many neutrophils and eosinophils in the muscle coat. The patient's recovery was smooth without any untoward event.

\section{Discussion:}

The first cases of secondary acute intestinal obstruction complicating primary acute appendicitis was described by Hotchkiss et al in 1901 and Hawkes in 1909., 2,3 Thereafter few additional studies were reported. ${ }^{9}{ }^{9}, 10 \mathrm{~A}$ systematic review of published articles from 1950 to 2016 was undertaken to define and characterize the various predisposing and precipitating risk factors, aetiology, pathogenesis, dominating presentations, clinical features and management by JG, Makama et al. ${ }^{1}$ Hawks in 1909 categorized the mechanisms into mechanical and septic appendicitis or a combination of both. Bhandari et al in 2009 categorized secondary acute intestinal obstruction following primary acute appendicitis into four types as adynamic, mechanical, strangulation and the one caused by mesenteric ischemia..$^{5}$ The mechanical one being seen as the commonest cause. ${ }^{5}$ They include (1) the appendix lying across the loops of bowel and get bound down to the posterior peritoneum at the tip and base by inflammatory adhesions. These are supported by Awale et al in 2015; (2) the appendix getting attached to the gut wall to form a floating band allowing one or multiple twists or kinks of small gut centering the appendicular band; (3) the tip of the appendix getting attached to the posterior abdominal wall or peritoneum or its base creating a hole or gap through which intestinal loops herniate leading to obstruction or strangulation. ${ }^{6}$ Assenza et al in 2005 reported such six cases. ${ }^{8}$ They described another mechanism as an appendix forming a simple or complex knot with any part of the small bowel. It is to be noted that the appendix is most commonly a mobile organ and can assume or take variable positions within the abdomen. Thence, when acutely inflamed, it tends to move and get adherent to other structures (like the ileum, the mesentery of the ileum, the caecum, the colon (the lying sigmoid loop or redundant transverse colon), the posterior peritoneum, even the right tubo-ovarian complex in females, or its tip to its base creating a mechanical factor. ${ }^{5}$ Another significant mechanism of secondary acute intestinal obstruction is by paralytic ileus. ${ }^{2,3}$ The inflammatory mediators released from the inflamed appendix and adjacent inflamed peritoneum possess inhibitory effects on the gut peristalsis. Here, hypokalemia resulting from excessive vomiting or toxins of sepsis or severe sepsis are equally important mechanisms as independent or contributing factors leading to secondary paralytic ileus complicating primary acute appendicitis. ${ }^{7-9}$ Gupta et al in 1969 was the first to report secondary acute intestinal obstruction in primary acute appendicitis resulting from ischaemia that may be from thrombo-embolic episodes due to acute inflammation in and around it including its mesentery the meso-appendix or simply an inflamed oedematous swollen appendix lying over a major branch of the ileocolic artery or one of its branches. ${ }^{6}$ Another review done by O'Donnell et al in 2009 described acute intestinal obstruction of some cases was due to a thromboembolic episodes of major branches of the ileal artery secondary to acute appendicitis. ${ }^{3}$ Bose et al in 1973 reported acute intestinal obstruction following primary acutely inflamed appendix as the latter wrapped round a loop of gut resulting in a strangulated closed loop obstruction. This is regarded by many surgeons as the fourth category. ${ }^{2,4}$ Predisposing factors (for developing secondary acute intestinal obstruction (duodenal, jejunal, ileal, large gut) include mobility and variable positions of the inflamed appendix, high mobility due to congenital malrotation of gut as described in 2000 by Zissen et al enhancing its adhesion or attachment to the other surrounding organs or their tissues, male gender (about 66.7\%, cause of which is yet to be explored), higher length of the appendix, higher length of the mesoappendix (facilitating its movement as described by Evers $\mathrm{MB}$ ), pre-ileal position of the appendix (compressing the ileum when the tip is eventually adhered down by adhesions to the posterior peritoneum as described by Bhandari L et al in 2009 and O'Donnell ME et al in 2009, recurrent appendicitisetc. ${ }^{2,3,8}$

The patient we had described and treated by surgery here in this case report was a male of twenty six years old who had presented with recurrent episodes of abdominal pain (simulating recurrent appendicitis) 
having a relatively floating non-compressing pelvic appendix. The inflamed floating gangrenous appendix easily adhered and involved the adjacent small intestinal loops. Transmigrated microbes, gangrenous appendix and sepsis were here significant contributory factors for secondary small intestinal obstruction complicating this acute attack on primary recurrent appendicitis. If the initial presenting clinical scenario are features of acute intestinal obstruction predominating over those of acute appendicitis, it is absolutely impossible to diagnose the primary pathology of acute appendicitis. Here in this case, we had got the history indicative of initial primary acute appendicitis. But as the features of intestinal obstruction at presentation predominated over those of acute appendicitis, we had diagnosed it as a case of small gut obstruction, keeping in mind that it might be a complication of maltreated acute appendicitis. The relevant history suggestive of primary acute appendicitis may not be always available and then the pre-operative diagnosis of the cause of acute intestinal obstruction would then be difficult, as our case was. Diagnosis then is usually made at per-operative findings. When the features of appendicitis predominate over those of acute intestinal obstruction, the intestinal obstruction may occur during the acute suppurartive phase of appendicitis. The obstruction may be due to paralytic ileus, mass effect, abdominal abscesses or thrombo-embolic phenomenon or sepsis or peritonitis following transmigration of microbes. The rarest clinical entity is when features of both conditions pre-dominate when it poses a definite challenge to pre-operative diagnosis. 4-D ultrasound, abdominal CT, MRI etc. nothing then can be definitely conclusively diagnostic. The presence of intra-abdominal exudate and mesenteric ischaemia seen by $\mathrm{CT}$ are here nonspecific. And delayed diagnosis and/or delayed intervention in the elderly increases the potential for higher morbidity and mortality. Emergency laparotomy through midline vertical incision gives better exposure, easy identification and the best treatment that includes immediate appendectomy and optimaladhesiolysis. If there is strangulated gut, viability is assessed. Early strangulation needs nothing more. In late cases, i.e., when gangrenous gut is there, segmental resection is followed by establishment of gut continuity by a suitable anastomotic procedure. Segmental resection is usually accompanied with decompression of distended loops. If the gangrenous bowel is close to the ileo-caecal junction, the appropriate treatment is a limited right hemicolectomy. Our case of intestinal obstructive feature had no mechanical predisposint factor and had no strangulated intestinal loop. When required, adequate peritoneal cleansing and toileting can minimize the morbidity and mortality. These additional procedures of resection and anastomosis cause increased duration of hospital stay, delay in convalescence and substantially increase in morbidity and mortality. These procedures are now can be accomplished by laparoscopically. ${ }^{1}$ Limitations in preoperative diagnosis of acute intestinal obstruction secondary to primary acute appendicitis are still existing as there is no confirmatory avant-garde imaging or other investigative aid other than awareness and careful history taking. Here we can realize and appreciate the clinical acumen by far as the more important one than laboratory aids in diagnosis of atypical presentation of acute appendicitis in the form of acute intestinal obstruction.

\section{Conclusion:}

Secondary acute intestinal obstruction complicating primary acute appendicitis needs to be borne in mind while dealing with acute abdomen. Careful clinical assessment and evaluation with adequate chronological history and a high index of suspicion are of paramount importance to arrive at a preoperative diagnosis. Even 4-D ultrasonogram, CT and MRI scans are not very often helpful. Pre-operative diagnosis may be contemplated with the help of raised inflammatory markers. Laparoscopy or laparotomy through midline vertical incision would be the best approach of choice whenever features of intestinal obstruction predominate, even if appendicitis is known to be the aetiologicalfactor.

Conflict of interest:

The authors have no conflict of interest.

\section{Acknowledgement:}

We are very much thankful to Prof.MizanurRahman, Department of Surgery,Monno Medical College, Manikganj for his inspiration in publishing the case report.

\section{References:}

1. Makama JG, Kache SA, Ajah LJ, Ameh EA.Intestinal obstruction caused by appendicitis: a systematic review. J West AfrColl Surg. 2017 Jul-Sep; 7(3): 94-115.

2. Awale L, Joshi BR, Rajbanshi S, Adhikary S. Appendiceal tie syndrome: A very rare complication of a common disease. World J Gastrointest Surg. 2015 Apr 27;7(4):67-70. 
3. Kareem H, Sebastian S, Jose T, Thomas V. Perforated appendicitis presenting with ileo-caecal ulceration and mechanical intestinal obstruction. Tropical Gastroenterology. 2008;29(2):105-6

4. Okello M, Kharono B, Mwaka E, Lock G. Appendico-ileal knotting mimicking adhesive bowel disease. Z Gastroenterol. 2016 Apr;54(4):316-8.

5. Sanjay H, Kamal M, Dougal B, Leslie B, Peter S. Acute appendicitis presenting as small bowel obstruction: two case reports. Cases Journal. 2009;2:9106-9163.

6. Bicer S, Celik A. Duodenal obstruction caused by acute appendicitis with intestinal malrotation in a child. Am J Case Rep. 2015 Aug 27;16:574-6.
7. Al-Reefia MA, Shukri N. Missed small bowel obstruction that complicated an acute appendicitis: a misdiagnosis: Case Report. Grand Rounds March 2013;13:36-41.

8. Evers MB.Small Intestine.Sabiston text book of surgery. Edited by: Townsend CM. 2008, Philadelphia: SAUNDERS, 2: 1296-18.

9. O'Donnell ME, Sharif MA.Small bowel obstruction secondary to an appendiceal tourniquet.Ir J Med Sci. 2009; 178: 101-5.

10. Assenza M, Ricci G. Mechanical small bowel obstruction due to an inflamed appendix wrapping around the last loop of ileum. G Chir.2005; 26: 261-6. 